\title{
Structure and Mechanical Properties of Nylon 6.12 \\ Prepared by Temperature Slope Crystallization I. \\ Crystallization of Oriented Spherulitic Textures.
}

Toshiho Yoshida, Tsutomu Asano, Masaaki Matsuura, Jun Kitabatake, Itaru Hatanaka, Kenji Seri, *Francisco J. Baltá Calleja and *Lily Giri

Faculty of Science, Shizuoka University, Ohya 836, Shizuoka 422, Japan *Instituto de Estructura de la Materia, CSIC, Serrano 119, 28006 Madrid, Spain

Corresponding Author: Prof. T. Asano,

Address: Faculty of Science, Shizuoka University, Ohya 836, Shizuoka 422, Japan.

FAX: +81-54-2380993 E-mail: sptasan@sci.shizuoka.ac.jp 


\begin{abstract}
Oriented spherulitic textures of a rod shaped nylon 6.12 sample were crystallized by the Temperature Slope Method. Crystallization conditions were compared by changing temperatures and growth rates. Three types of textures : negative spherulites, positive spherulites and spherulitic aggregates were observed by this method. The negative textures appeared when the growth rate less than $0.1 \mathrm{~mm} /$ hour. Crystalline orientation and mechanical properties of the textures were investigated by X-ray diffraction and microhardness measurements, respectively. The hydrogen bonded (010) planes were perpendicular to the growth direction in the negative spherulite, while they were parallel to the growth direction in the positive spherulite. In the spherulitic aggregates, the b-axis is parallel to the growth direction, where the $(010)$ planes form roughly an angle of $45^{\circ}$ with the growth direction. Due to the orientation of the hydrogen bonded planes, the negative texture exhibits an anisotropy, with hardness values of $106 \mathrm{MPa}$ and $137 \mathrm{MPa}$ when mearsured perpendicular or parallel to the growth direction, respectively.
\end{abstract}

Running Title: $\quad$ Oriented spherulitic textures of nylon 6.12 . 


\section{INTRODUCTION}

Morphology of polymeric spherulites crystallized from the melt has been widely investigated in recent years $[1,2,3]$. Most of these studies were performed isothermally crystallized samples. Fujiwara et al. reported oriented spherulitic textures prepared by temperature slope crystallization (TSC) $[4,5]$. In these investigations, spherulitic textures were solidified from the melt under a steep temperature gradient. Using the uniaxially oriented $\beta$-phase polypropylene texture crystallized by the TSC method, an anisotropic behavior in the deformation process has been observed $[6,7,8]$.

The mechanical properties of nylon are of special interest because of the behavior of the hydrogen bonded planes, which may show a different deformation mechanisms from those of polyethylene or polypropylene. Within this context, Yoshida et al. have tried to apply the TSC method to nylon samples thicker than $0.5 \mathrm{~mm}$ [9].

Lovinger reported the directional solidification of even-even nylons such as nylon $66,6.10$ and 6.12 , using film thickness of 20-200 $\mu \mathrm{m}$, sandwiched between slide glasses [10]. By means of directional solidification, it is possible to distinguish the morphological diversity of nylons, and discuss the specific morphology and orientation of the various types of textures appearing in eveneven nylons [11].

In a preceding paper the rolling deformation of nylon 6.12 and the orientation of the hydrogen bonded planes, with respect to the surface of rolling was investigated by means of microhardness measurements [12]. Recent studies have widely evidenced the existing relationship between microhardness and morphology of polymers [13,14]. Microhardness, in addition, has been shown to be capable to reveal polymorphic changes in polymers [15]. Recent results have also shown that hardness determination is a promising technique for the microstructural investigation of multicomponent blends and can provide information on the degree of interpenetration of the blend components [16].

In the present paper, the TSC method was applied to a thick rod sample of nylon 6.12 and morphology is investigated by changing the crystallizing conditions. The orientation and mechanical properties of three types of spherulitic textures: negative spherulites, positive spherulites and 
spherulitic aggregates of nylon 6.12 are analyzed by means of $X$-ray diffraction and microhardness measurements.

\section{EXPERIMENTAL}

\section{1) Sample Preparation}

A sample of poly(hexamethylene dodecane diamide), generally known as nylon 6.12 was used in this work. The sample was purchased from Scientific Polymer Products INC. with the sample number of \#313, and the specific gravity of 1.06. For removing water, the sample pellets were vacuum dried at $100^{\circ} \mathrm{C}$ for one week in a preliminary treatment. Thereafter, the sample was melt under vacuum and spun to a rod with diameters about $1 \mathrm{~mm}$. Then, it was put into a thin glass tube with $2 \mathrm{~mm}$ in diameter, $0.2 \mathrm{~mm}$ in thickness. Finally, the sample glass tube was sealed by gas fire after vacuum drying at room temperature for $30 \mathrm{~min}$.

The crystalline structure of nylon 6.12 is known to be triclinic with $a=4.97 \AA, b=5.37 \AA, c=25.1$ $\AA, \alpha=51.1^{\circ}, \beta=77.1^{\circ}, \gamma=63.5^{\circ}[9]$. Nylon 6.12 exhibits three kinds of spherulites: the positive spherulites, negative spherulites and spherulitic aggregates. All these textures have the same crystalline structure, although they exhibit different crystal orientations. The thermal analysis of the original sample shows a melting temperature $\left(\mathrm{T}_{\mathrm{m}}\right)$ of $218^{\circ} \mathrm{C}$ when the sample was heated at 0.5 ${ }^{\circ} \mathrm{C} / \mathrm{min}$.

\section{2) Temperature Slope Crystallization (TSC)}

Crystallization under temperature gradient was developed by Fujiwara et. al $[4,5]$. In the present experiment, nylon 6.12 sample was crystallized by the specially designed apparatus using a temperature slope shown in Fig.1a. Two heat reservoirs ( a heater A and a cooler B ) were maintained at temperatures $T_{A}\left(>T_{m}\right)$ and $T_{B}\left(<T_{m}\right)$, respectively. A temperature slope was prepared using the heater and the cooler with a gap dimension of $2.0 \mathrm{~mm}$. The heater temperature was controlled to the setting temperature $\left(\mathrm{T}_{\mathrm{A}}\right)$, lying between $350^{\circ} \mathrm{C}$ and $250^{\circ} \mathrm{C}$. The cooler was water cooled at a temperature of $15^{\circ} \mathrm{C}$. The temperature slope was measured by a thermo-couple inserted in the sample tube. Fig.1b shows the temperature gradient measured with a thermo-couple moving 
along the temperature slope from the heater to the cooler with a velocity of $0.3 \mathrm{~mm} / \mathrm{hr}$. The maximum temperature gradient was $45.6^{\circ} \mathrm{C} / \mathrm{mm}$ when $\mathrm{T}_{\mathrm{A}}=280^{\circ} \mathrm{C}$.

Nylon 6.12 sample was mounted on the TSC apparatus. When the specimen was moved with a constant speed $U$ along the temperature slope, stationary lamellar growth was observed in the form of a melt-solid interface at a fixed position between A and B. Here, lamellar growth rate $G$ balanced with the moving speed $U$ of the specimen. In this experiment, the crystallization rate $G$ was changed from 0.07 to $1.0 \mathrm{~mm} / \mathrm{hour}$.

\section{3) Microscopy and X-ray Diffraction}

The rod sample crystallized under the temperature slope was cut into thin film slices by a microtome. The sphrulitic textures were observed by a polarized microscope. Crystalline structure and orientation were studied by wide angle $X$-ray scattering (WAXS) patterns taken with a Rigaku RU-300 X-ray generator. The super structure (lamellar structure) was also determined by small angle X-ray scattering (SAXS) with Ni filtered CuK $\alpha$ radiation. The WAXS and the SAXS were measured with incident $\mathrm{X}$-rays perpendicular to the growth direction.

\section{4) Microhardness Measurement}

The microhardness $(\mathrm{H})$ was measured at room temperature using a Leitz tester adapted with a square based pyramid indenter. The contact area was obtained by measuring the residual image of the projection of indented area. A loading cycle of 0.1 min was used to minimize the creep, and different loads $0.15,0.25,0.5$ and $1 \mathrm{~N}$ were employed. The microhardness value was obtained using the equation:

$$
\mathrm{H}=\mathrm{KP} / \mathrm{d}^{2} \text {, }
$$

where $\mathrm{d}$ is the impression diagonal, $\mathrm{P}$ the contact load applied and $\mathrm{K}$ a geometrical factor equal to 1.854 . 


\section{RESULTS AND DISCUSSION}

\section{1) Best Condition of TSC}

The temperature slope crystallization for nylon 6.12 was thoroughly examined by changing the heater temperature $\left(T_{A}\right)$, the gap distance and the crystallization rate $(G)$. Yoshida et al. previously obtained a well oriented positive texture using values of $\mathrm{T}_{\mathrm{A}}=320^{\circ} \mathrm{C}$ and $\mathrm{G}=1 \mathrm{~mm} / \mathrm{hr}$ [9]. The spherulitic aggregates were developed by a slower crystallization rate around $0.3 \mathrm{~mm} / \mathrm{hr}$. In the crystallization of even-even nylon for thin films of 20-200 $\mu \mathrm{m}$, Lovinger reported that the negative spherulite was grown when $T_{A}=400^{\circ} \mathrm{C}$ and $\mathrm{G}=0.1$ to $0.3 \mathrm{~mm} / \mathrm{hr}[10,11]$. In our case, the crystallization condition was shifted due to the thicker diameter of the sample $(2 \mathrm{~mm})$ than the case of Lovinger.

Crystallization of the three types of spherulites was obtained by changing $T_{A}$ and $G$. The crystallization conditions were qualitatively classified by optical microscopy ( texture orientation, number of nuclei ) and by WAXS patterns ( arc length of 100 and 010 reflections ). The results are summarized in Table 1. The best condition for the negative spherulites appeared at a crystallization rate $(G)$ lower than $0.1 \mathrm{~mm} / \mathrm{hr}$. Orientation and a variety of textures were affected by the $T_{A}$ and $\mathrm{G}$. With increasing growth rate $\mathrm{G}$, the positive spherulites appeared predominantly accompanied by spherulitic aggregates.

\section{2) Spherulite Textures under the Polarizing Microscope}

When the polymer rod was crystallized at the rate of $0.07 \mathrm{~mm} / \mathrm{hr}$, negative spherulites primarily appeared at the crystallizing boundary. The oriented negative texture continued for several tens of hours producing a negative region of $1-2 \mathrm{~mm}$ in the sample. After that, the nuclei of the spherulitic aggregates appeared. Then, the negative texture soon disappeared and changed into the form of aggregates because of the difference of the intrinsic growth rates. Following the aggregate region of 1-2 $\mathrm{mm}$, the positive spherulites developed producing a positive region. These three textures were easily distiguished under the polarizing microscope. 
In Fig. 2(a), the negative texture is looking rather smooth whereas the aggregates show a very rough appearance. In this case, the stationary growth of the negative spherulite might be disturbed by an external oscillation of the crystallization apparatus or by a fluctuation of the heater temperature. Once the nuclei appear, the spherulitic aggregates developed rapidly and soon covered the whole space of the sample.

Features of the oriented positive spherulite are shown in Fig.2(b). Some parts of the texture includes a large number of nuclei. For the higher crystallization rate, the positive texture is followed by the aggregates as shown in Fig.2(c).

\section{3) Crystalline Orientation measured by X-ray Diffraction}

The diffraction patterns of the three spherulitic textures are illustrated in Fig.3. The patterns show very broadly arced rings in the three textures. However, the orientation of the hydrogen bonded (010) plane is comparable with the results of Lovinger [11]. From the direction of the maximum intensity of 010 reflection in Fig.3(c), the positive spherulite has its (010) planes parallel to the growth axis. Similarly to Lovinger's results, the negative spherulite, in our case, also has the (010) planes perpendicularly oriented to the growth direction $G$. In spite of their rough appearance in the polarized microscope, the spherulitic aggregates show a well defined orientation in the WAXS pattern of Fig.3(b). In this case, the hydrogen bonded (010) planes are oriented about $45^{\circ}$ to the growth direction. In Fig.4, the possible orientations which were discussed by Lovinger for eveneven nylon and the expected WAXS patterns for the three textures are comparatively presented.

From these observations, the three spherulitic textures observed in our TSC method can be summarized as follows:

(a) Negative spherulites : the $b^{*}$-axis is parallel, i.e. the hydrogen bonded $(010)$ plane is perpendicular to the growth direction $\mathbf{G}$.

(b) Spherulitic Aggregates : the b-axis is parallel to the growth direction, where the $(010)$ plane makes roughly $45^{\circ}$ with the growth direction $\mathbf{G}$.

(c) Positive spherulites : the a-axis is parallel to the growth direction, where the $b^{*}$-axis is 
perpendicular, i.e. the $(010)$ plane is parallel to the growth direction $\mathbf{G}$.

From the SAXS patterns of the three spherulites, crystalline lamellae are inclined and distributed in the positive and negative spherulites, whereas the lamellar normal is perpendicular to the growth direction in the spherulitic aggregates.

By considering the triclinic unit cell, the crystalline (001) plane of the negative crystal is inclined from $\mathbf{G}$. As a result, the lamellar surface is inclined about $40^{\circ}$ from $\mathbf{G}$, which induces surface roughness of the negative texture as depicted in Fig. 5, and also gives rise to the diffuseness in the SAXS pattern (Fig. 3d). However, the average orientation of the lamellar normal is perpendicular to $\mathrm{G}$ in the three spherulitic textures.

\section{4) Microhardness measurement of the three spherulitic textures}

The mechanical properties were studied through determination of the microhardness $\mathrm{H}$ in the three spherulitic textures. The results are summarized in Table 2. The surface hardness values of the sample crystallized by TSC were $79-91 \mathrm{MPa}$. These values were lower than those obtained with the oriented nylon 6.12 prepared by roll deformation (>100 MPa) [12]. In order to remove the influence of water, we kept the sample in vacuum at $35^{\circ} \mathrm{C}$ for $48 \mathrm{hrs}$, i.e. below the glass transition temperature. The results of the microhardness, measured immediately after such drying treatment, showed a remarkable hardening. After drying, the $H$ values increased more than 100 $\mathrm{MPa}$. This result shows that humidity reduces substantially the mechanical properties of the sample. Due to the hydrogen bonding between water and nylon molecules, number of the hydrogen bonds in the nylon crystal diminishes, causing a decrease of $\mathrm{H}$.

In order to measure the microhardness anisotropy, the $\mathrm{H}$ values of the side-face and end-face were compared as shown in Table 2. In the negative spherulite, the $\mathrm{H}$ value of the end-face, perpendicular to the growth direction $(\mathbf{G})$, is higher than the side-face parallel to $\mathbf{G}$. The results are reasonable if we consider the orientation of the hydrogen bonded (010) plane which is perpendicular to the growth direction $\mathbf{G}$. 


\section{CONCLUSIONS}

1. Crystallization by the TSC method is studied using a rod shaped nylon 6.12 sample with $2 \mathrm{~mm}$ in diameter. Crystallization conditions for three types of textures: negative spherulites, positive spherulites and spherulitic aggregates are examined, where the negative one is developed when the crystallization rate lower than $0.1 \mathrm{~mm} / \mathrm{hour}$.

2. By using polarized microscopy and $X$-ray diffraction measurements, the orientation of the three spherulitic textures is characterized as follows:

(a) Negative spherulites : the $b^{*}$-axis is parallel, i.e. the hydrogen bonded (010) planes are perpendicular to the growth direction $\mathbf{G}$.

(b) Spherulitic Aggregates : the b-axis is parallel to the growth direction, where the $(010)$ planes form roughly an angle of $45^{\circ}$ with the growth direction $\mathbf{G}$.

(c) Positive spherulites: the a-axis is parallel to the growth direction, where the $(010)$ planes are parallel to the growth direction $\mathbf{G}$.

3. Microhardness measurements show a value of $113 \mathrm{MPa}$ in the positive texture and of $128 \mathrm{MPa}$ in the spherulitic aggregates. Due to the orientation of the hydrogen bonded planes, the negative texture exhibits an anisotropy, with $\mathrm{H}$ values of $106 \mathrm{MPa}$ and $137 \mathrm{MPa}$ when measured perpendicular or parallel to the growth direction, respectively.

\section{ACKNOWLEDGMENTS}

The authors express our deep gratitude to Prof. Y. Fujiwara for his warm instructions and suggestions to the TSC method. Thanks are due to DGICYT, Spain (Grant PB94-0049) for the generous support of this investigation. 


\section{REFERENCES}

1. D.C.Bassett, in "Self Order and Form in Polymeric Materials", Eds. A. Keller, M. Warmer, A.H. Windle, Chapman and Hall, London, p27 (1995)

2. P.J. Barham, in "Materials Science and Technology. A Comprehensive Treatment" vol.12, Ed.

E.L. Thomas. Structure and Properties of Polymers. V.C.H. Weinheim (1993).

3. J. Varga in "Polypropylene. Structure, blends and composites" vol. 1, Structure and Morphology. Ed. J. Larger-Kocsis, Chapman and Hall, London, p56 (1993).

4. Y. Fujiwara, Kolloid Z. -Z Polym. 226, 135 (1968)

5. K. Tanaka, T. Seto and Y. Fujiwara, Rep. Progr. Polym. Phys. Jpn. 6, 285 (1963)

6. T. Asano and Y. Fujiwara, Polymer, 19,99 (1978)

7. T. Asano, Y. Fujiwara and T. Yoshida, Polymer J. 11,383 (1979)

8. T. Yoshida, Y. Fujiwara and T. Asano, Polymer, 24, 925 (1983)

9. T. Yoshida and N. Miyshita, Rep. Fac. Sci. Shizuoka Univ., 21, 31 (1987)

10. A.J. Lovinger, J. Appl. Phys., 49 , 5003 (1978)

11. A.J. Lovinger, J. Appl. Phys., 49 , 5014 (1978)

12. F. J. Baltá Calleja, L. Giri, T. Asano and T. Yoshida, Rep. Fac. Sci. Shizuoka Univ. , 28, 53 (1994)

13. C. Santa Cruz, F.J. Baltá Calleja, H.G. Zachmann, N. Stribeck and T. Asano, J. Polymer Sci., B29, 819 (1991)

14. F.J. Baltá Calleja, C. Santa Cruz and T. Asano, J. Polym. Sci., 31, 557 (1993)

15. F. J. Baltá Calleja, J. Martinez Salazar and T. Asano, J. Mater. Sci. Lett., 7, 165 (1988)

16. F. J. Baltá Calleja, C. Santa cruz, C. Sawatari and T. Asano, Macromolecules, 23, 5352 (1990) 


\section{Table 1}

Selected crystallization conditions of the TSC method. Orientation was determined qualitatively by $\mathrm{X}$-ray diffraction and polarized microscopy :

(+) good orientation in the texture,

(-) bad orientation or no orientation.

\begin{tabular}{ccccc}
\hline Heater & Growth Rate & \multicolumn{2}{c}{ Orientation of the Textures } \\
\hline Temperature & $(\mathrm{G})$ & Negative & Aggregates & Positive \\
$\left({ }^{\circ} \mathrm{C}\right)$ & $(\mathrm{mm} / \mathrm{hr})$ & & & \\
\hline 320 & 0.197 & - & - & + \\
310 & 0.179 & - & + & + \\
310 & 0.125 & - & - & + \\
307 & 0.121 & - & - & - \\
305 & 0.115 & - & - & - \\
280 & 0.109 & - & + & + \\
275 & 0.085 & + & + & + \\
265 & 0.072 & + & + & + \\
255 & 0.073 & + & + & + \\
\hline
\end{tabular}




\section{Table 2}

Microhardness ( $\mathrm{H}$ ) of the three spherulitic textures obtained by the TSC method. The anisotropy in $\mathrm{H}$ was compared in the sample surface parallel to $\mathrm{G}$ (Side-face) and perpendicular to $G$ (End-face).

\begin{tabular}{lcc}
\hline \multicolumn{1}{c}{ Textures } & Microhardness & $(\mathrm{MPa})$ \\
\hline & Side-face & End-face \\
Negative Spherulite & $106(* 79)$ & 137 \\
Spherulitic Aggregates & $128(* 91)$ & 122 \\
Positive Spherulite & $113(* 82)$ & 111 \\
\hline
\end{tabular}

* Measurements without drying treatment. 


\section{FIGURE CAPTIONS}

Figure 1 Method of the temperature slope crystallization (TSC).

(a) Schematic representation of the apparatus. The temperature slope was made between the heater A and the cooler B. (b) Temperature gradient observed.

Figure 2 Oriented textures observed under the polarizing microscope; (a) negative spherulites (bottom), spherulitic aggregates (top), (b) positive spherulites, (c) positive spherulites (bottom), spherulitic aggregates (top).

Figure 3 Top: WAXS of (a) negative spherulites, (b) spherulitic aggregates, (c) positive spherulites. Bottom: SAXS of (d) negative spherulites, (e) spherulitic aggregates, (f) positive spherulites. Growth direction : G

Figure 4 Crystalline orientations for the three spherulitic textures and the expected WAXS patterns: (a) negative spherulites, (b) spherulitic aggregates and (c) positive spherulites.

Figure 5 Orientation of the hydrogen bonded (010) planes and surface roughness in (a) negative texture, (b) positive texture. The dotted lines indicate the average lamellar texture. 
(a)

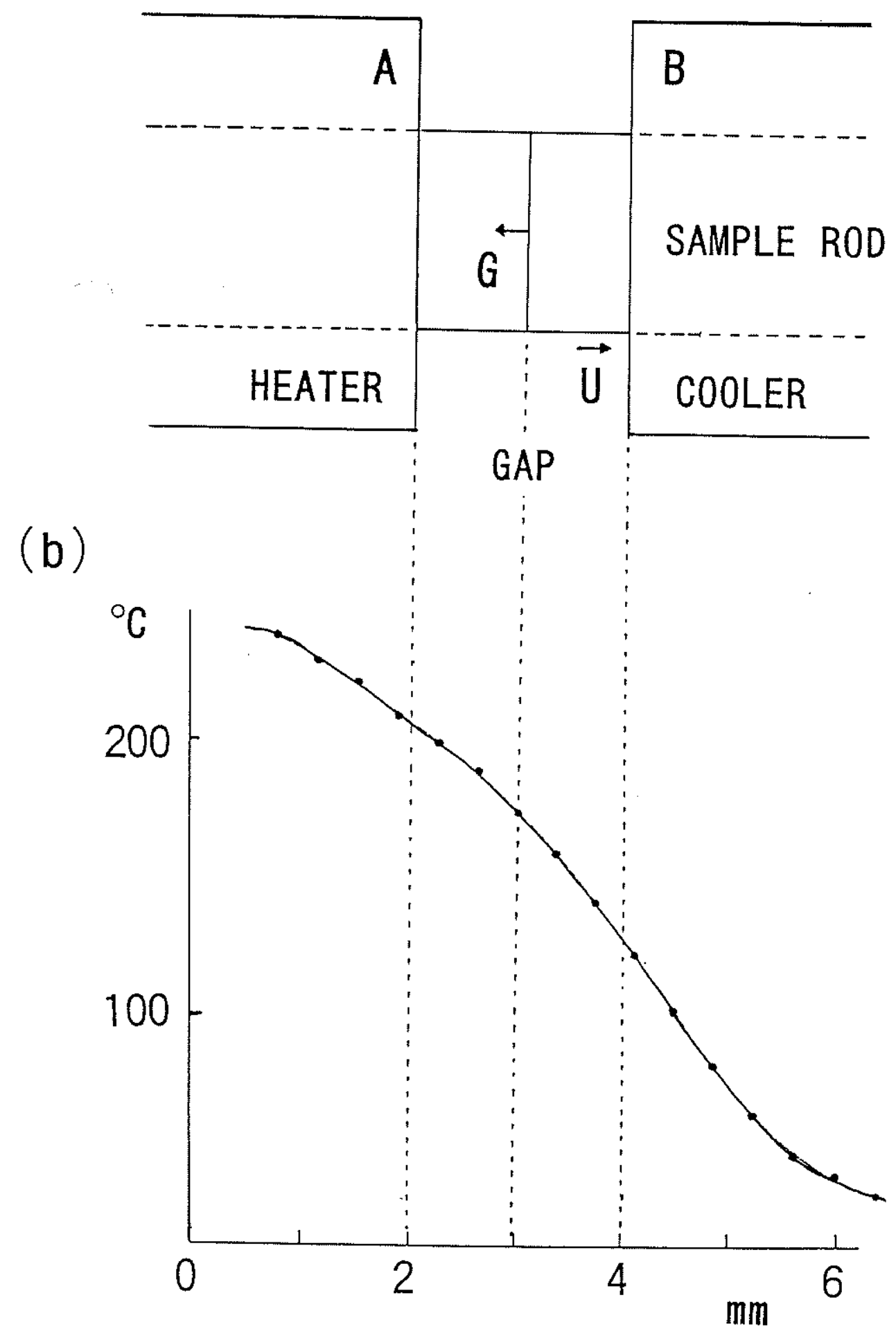

Fig.1 

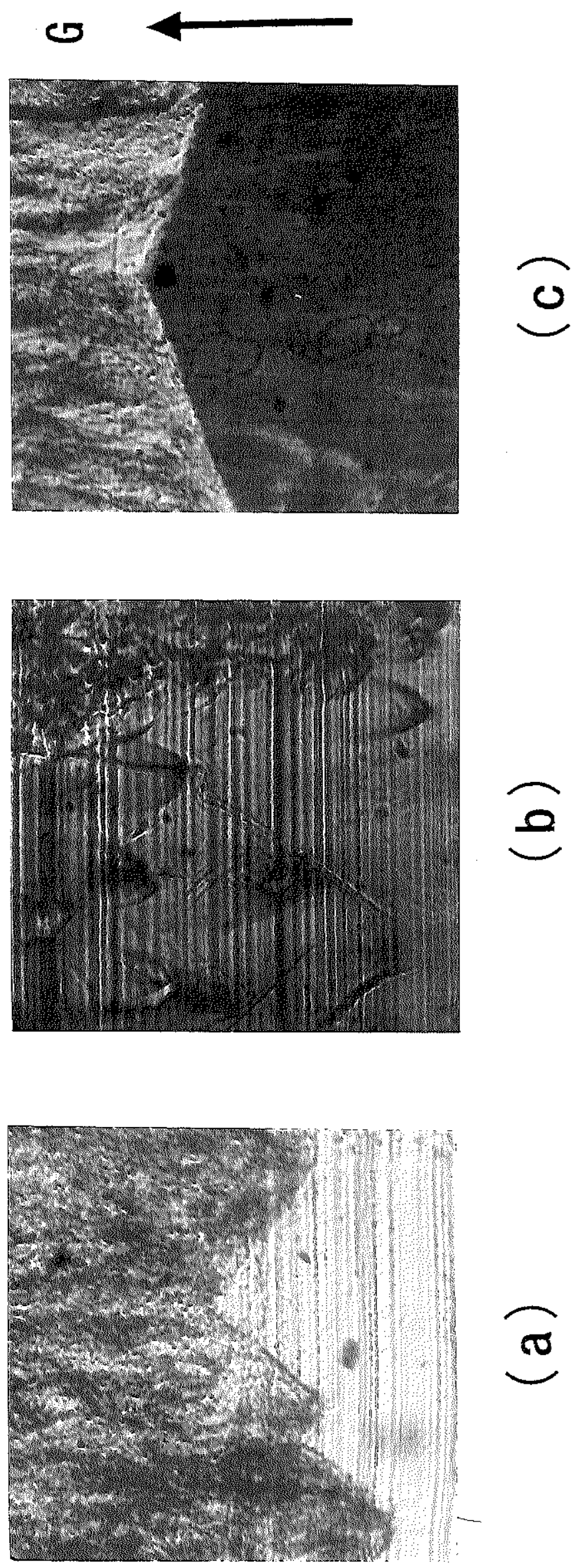

Fig.2 

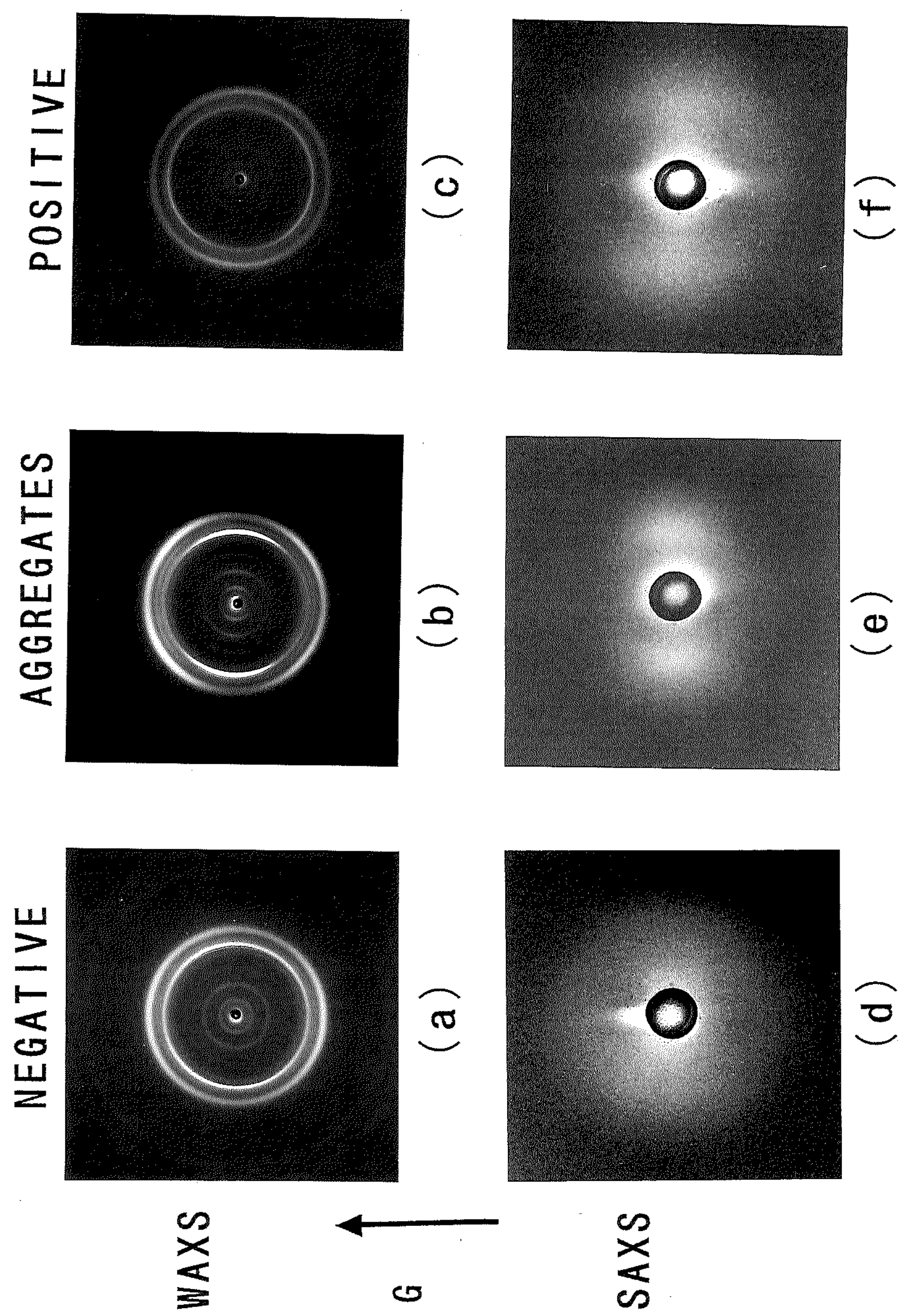

Fig.3 


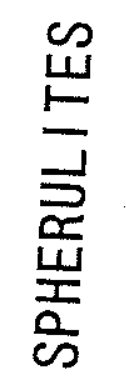

崖

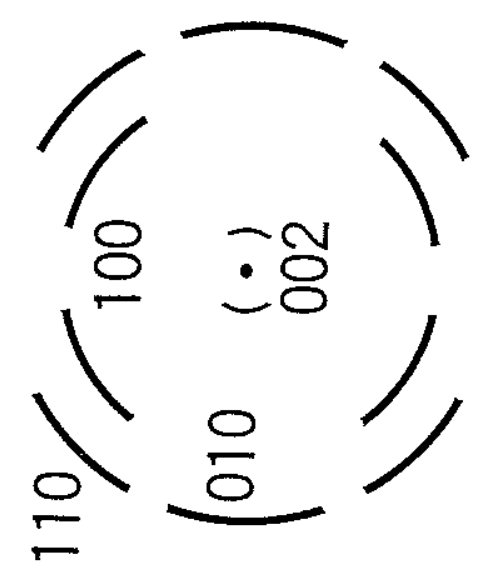

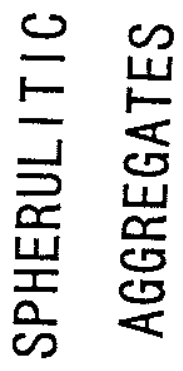
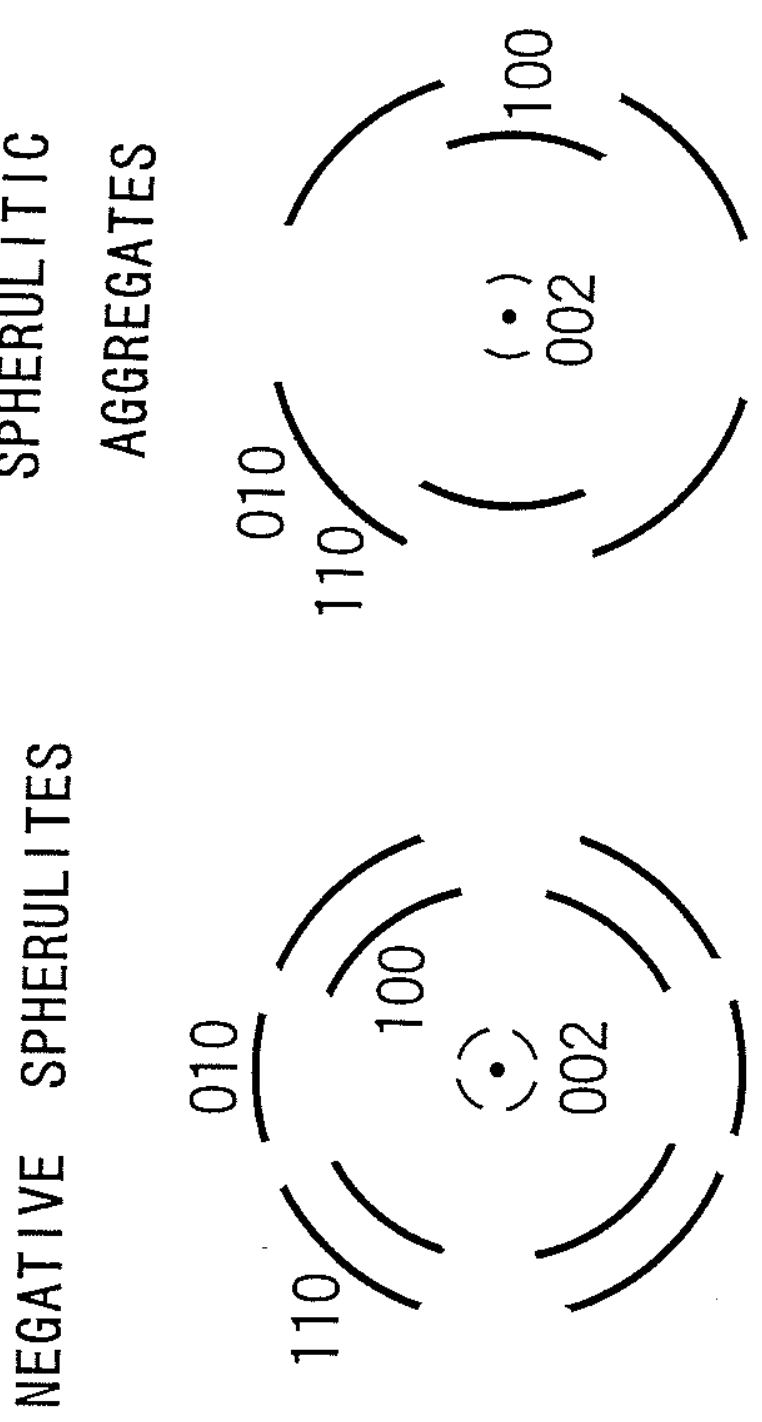
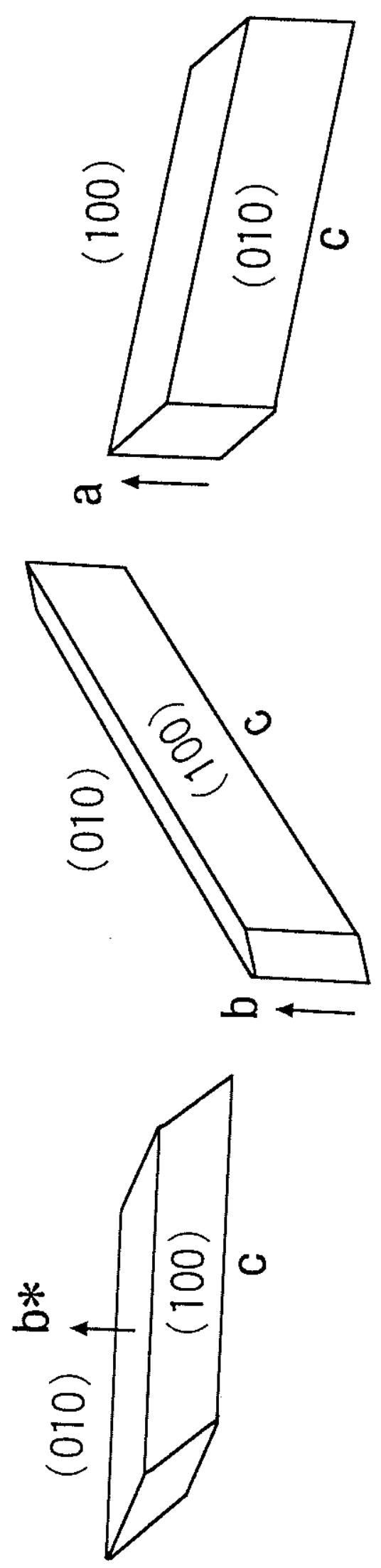

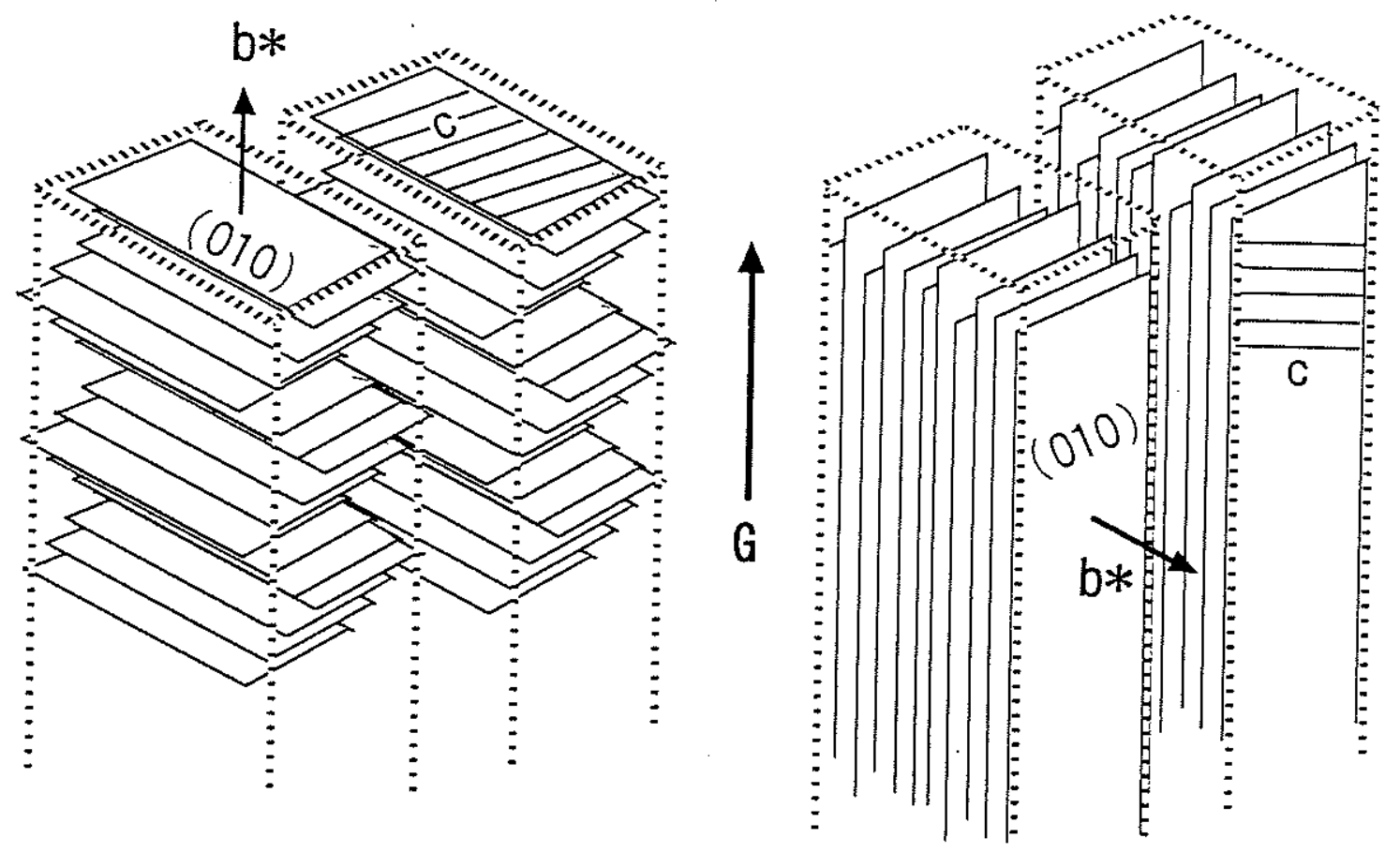